\title{
Impulsive Consensus Control for Complex Dynamical Networks with Non-identical Nodes and Coupling Time-Delays
}

\author{
Bin Liu, David J. Hill
}

\begin{abstract}
This paper investigates the problem of global consensus between a complex dynamical network (CDN) and a known goal signal by designing an impulsive consensus control scheme. The dynamical network is complex with respect to the uncertainties, non-identical nodes and coupling timedelays. The goal signal can be a measurable vector function or a solution of a dynamical system. By utilizing Lyapunov function and Lyapunov-Krasovskii functional methods, robust global exponential stability (RGES) criteria are derived for the error system, under which global exponential consensus is achieved for the complex dynamical networks. These criteria are expressed in terms of LMIs and algebraic inequalities. Thus, the impulsive controller can be easily designed by solving the derived inequalities. Meanwhile, the estimations of the consensus speed rate for global exponential consensus is also obtained. One example with numerical simulations is worked out for illustration.
\end{abstract}

\section{INTRODUCTION}

Synchronization of chaotic systems and its potential applications to secure communication has been an active research area since the 1990's. Numerous methods have been developed for chaos synchronization (see, for example, [1-11]). Recently, synchronization of complex dynamical networks (CDNs) is also reported in the literature (see, for example, [12-27]). The complex dynamical network consists of coupled nodes, which are usually dynamical systems. There have been proposed several approaches for synchronization of a CDN, for example, feedback control synchronization (see, for example, [22-23]), adaptive synchronization [20], synchronization based on the invariance principle [10], stateobserver-based approach [26], and impulsive synchronization [34], etc.

It has been noticed that the complex dynamical networks (CDNs) studied in the literature have the following limitations: (i) the coupled nodes have the same dynamics; (ii) by using the linearization technique and matrix eigenvalue method, most synchronization criteria are local; (iii) uncertainty and time delays which are common in practical CDNs have not been studied fully, although there are published results $([8,15,20,34])$ which study the robust synchronization problem with respect to uncertainties, and some ([2124]) deal with a single constant time delay. Uncertainties often occur due to the uncertainties of parameters, modeling

This work was supported by the Australian Research Council Discovery Project Scheme (No. FF0455875 and No. DP0881391).

B. Liu is with the Department of Information Engineering, The Australian National University, ACT 0200, Australia. Bin. Liu@anu. edu. au

D. J. Hill is with the Department of Information Engineering, The Australian National University, ACT 0200, Canberra, Australia. David.Hilleanu.edu.au mismatches, measurement errors, approximations, channel noises etc. And time delays occur commonly due to the congestion of the network traffic and the fact that the switching and spreading speed of the hardware and circuit implementation is finite. Moreover, the time delays presented in many real synchronization schemes are difficult to know a priori and they are in the form of multiple time delays and time-varying.

The CDNs with non-identical nodes represent more general and practical networks than the models typically studied in the literature. Moreover, to the best of our knowledge, no literature has been published for the consensus problem between CDNs and a known goal function. However, allowing different dynamics of nodes in a CDN brings difficulties in achieving consensus. If the uncertainty and time delays occur simultaneously in a CDN with non-identical nodes, and consensus is to be achieved to a known goal function, it will be much more difficult to use previous synchronization control schemes, specially for the global consensus problem. Hence, there is a need to study new consensus control schemes which can achieve the objective.

In this paper, we propose an impulsive consensus control scheme for the consensus problem between CDNs and the known signal. In this control scheme, the control signal is designed to input into the $\mathrm{CDN}$ as follows: at impulsive instances, the impulse signal is input into the nodes, and at other times, the signal containing the goal signal is input into the nodes. Hence, this control scheme is a type of impulsive control scheme. Impulsive control arises naturally from a wide variety of applications, such as drug administration, spacecraft control, inspection processes in operations research, native forest ecosystems management, just to name a few. Based on the stability theory of impulsive systems (see [28-30, 35-38], and references therein), the impulsive control method (see [31-33, 39] and references therein) provides greater prospect for solving many problems that are basically defined by continuous dynamical systems, but on which only discrete-time actions are exercised. An essential benefit of the impulsive control approach may be derived from the fact that such controls are typically simpler and cheaper to implement. In [9], impulsive control was first introduced to synchronize chaotic systems. Since then, significant progress has been made in impulsive synchronization of chaotic systems, see [11] and the references therein. Recently, impulsive synchronization for CDNs was also reported in [34]. The theory and experiments have proved that the impulsive synchronization scheme for chaotic systems or CDNs (with identical nodes) has good robustness against uncertainties and can achieve 
global synchronization.

The aim of this paper is to study the global exponential consensus problem for CDNs and a known goal function by using an impulsive consensus control scheme. The model of CDNs consists of non-identical nodes, uncertainties, and coupling time delays. Here, the "uncertainties" means the uncertainty of parameters, which take values in some intervals. The exponential consensus scheme has an obvious advantage over other consensus schemes, in which the consensus speed and consensus time can be estimated easily. By utilizing the Lyapunov function and Lyapunov-Krasovskii functional ([4041]) methods, robust global exponential stability (RGES) results for delay error systems shall be established, and then we shall derive several criteria under which the global exponential impulsive consensus (GEIC) is achieved for the CDNs. These criteria are expressed in terms of LMIs and algebraic inequalities. Thus, the conditions of consensus are easy to be tested. Moreover, the solutions of the LMIs and algebraic inequalities give rise directly to impulsive controllers for a CDN under which GEIC is achieved.

\section{Preliminaries And Problem Formulation}

Let $R^{n}$ denote the $n$-dimensional Euclidean space. Let $R_{+}=[0,+\infty), \mathbb{Z}=\{0,1,2, \cdots$,$\} , and \|\cdot\|$ be the Euclidean norm in $R^{n}$. Let $I$ be the identity matrix, and matrix $X>$ $(\geq,<, \leq) 0$ means that $X$ is a symmetric positive definite (positive semi-definite, negative definite, negative semidefinite) matrix. Denote by $\lambda_{\max }(\cdot)\left(\lambda_{\min }(\cdot)\right)$ the maximum (minimum) eigenvalue of matrix $(\cdot)$.

Consider the uncertain $\mathrm{CDN}$ consisting of $N$ non-identical nodes ( $n$-dimensional dynamical systems) with coupling time-delays:

$\dot{x}_{i}=f_{i}\left(t, x_{i}\right)+g_{i}\left(x_{1}\left(t-h_{i}\right), \cdots, x_{N}\left(t-h_{i}\right)\right), i=1, \cdots, N$,

where $x=\left(x_{1}^{T}, x_{2}^{T}, \cdots, x_{n}^{T}\right)^{T} \in R^{n N}, x_{i}=\left(x_{i 1}, x_{i 2}, \cdots, x_{i n}\right)^{T} \in$ $R^{n}$ represents the state vector of the $i$ th node; $f_{i}: R_{+} \times R^{n} \rightarrow$ $R^{n}$ is a smooth vector function; $g_{i}: R^{n N} \rightarrow R^{n}$ is a smooth vector function representing the coupling of the $i$ th node; $h_{i}$ is some nonnegative constant which represents the timedelay of the signal transmitted from the network to the $i$ th node, where the coupling time-delay $h_{i}$ satisfies $0 \leq h_{i} \leq \tau$ for some constant $\tau>0$ and $i=1,2, \cdots, N$. We call the system (1) an uncertain network based on the fact that there are uncertainties in functions $f_{i}, g_{i}, i=1,2, \cdots, N$. In this paper, we make the following assumptions:

Assumption 2.1. Assume that $f_{i}\left(t, x_{i}\right)=A_{i} x_{i}+\tilde{f}_{i}\left(t, x_{i}\right)$, where $A_{i} \in R^{n \times n}$ is an interval matrix with $A_{i} \in N\left[A_{i_{1}}, A_{i_{2}}\right]$, where $N\left[A_{i_{1}}, A_{i_{2}}\right]=\left\{\left(a_{i j}\right) \in R^{n \times n}: u_{i j} \leq a_{i j} \leq v_{i j}\right\}$ for known matrices $A_{i_{1}}=\left(u_{i j}\right)_{n \times n}$ and $A_{i_{2}}=\left(v_{i j}\right)_{n \times n}$, and function $\tilde{f}_{i}$ satisfies $\left\|\tilde{f}_{i}\left(t, s_{1}\right)-\tilde{f}_{i}\left(t, s_{2}\right)\right\| \leq L_{i}\left\|s_{1}-s_{2}\right\|$, for some positive constant $L_{i}>0$ and for all $t \in R_{+}$.

It should be noticed that when the network (1) achieves consensus, namely, when the states $x_{1}(t)=\cdots=x_{N}(t)=$ $s(t)$ as $t \rightarrow \infty$, the coupling terms should vanish: i.e., $g_{i}(s, s, \cdots, s)=0, i=1,2, \cdots, N$. Thus, we give the following assumption on function $g_{i}$ :
Assumption 2.2. Assume that $g_{i}\left(x_{1}, \cdots, x_{N}\right)=\sum_{j=1}^{N} B_{i j} x_{j}+$ $\tilde{g}_{i}\left(x_{1}, \cdots, x_{N}\right)$, where $B_{i j} \in R^{n \times n}$ is an interval matrix with $B_{i j} \in N\left[B_{i j_{1}}, B_{i j_{2}}\right]$, where $B_{i j_{1}}, B_{i j_{2}}$ are known matrices, and matrices $B_{i j}$ and function $\tilde{g}_{i}$ satisfy:

$$
\sum_{j=1}^{N} B_{i j}=0, \quad i=1,2, \cdots, N
$$

and $\tilde{g}_{i}(s, s, \cdots, s)=0$, and for some constants $M_{i j} \geq 0$,

$$
\left\|\tilde{g}_{i}\left(x_{1}, \cdots, x_{N}\right)-\tilde{g}_{i}\left(y_{1}, \cdots, y_{N}\right)\right\| \leq \sum_{j=1}^{N} M_{i j}\left\|x_{j}-y_{j}\right\| .
$$

Problem formulation: Let $s(t)$ be a given measurable smooth vector function satisfying $s(t) \in R^{n}$ for any $t \in R_{+}$. The aim of this paper is to design an impulsive hybrid control scheme for the CDN (1) such that the consensus among the node states $x_{i}(t)(i=1,2, \cdots, N)$ and goal $s(t)$ can be achieved.

Consider the CDN (1) under impulsive consensus control:

$$
\dot{x}_{i}=f_{i}\left(t, x_{i}\right)+g_{i}+u_{i}\left(t, x_{i}, s\right), \quad i=1,2, \cdots, N,
$$

where $\left\{u_{i}\left(t, x_{i}, s\right), i=1,2, \cdots, N\right\}$ is the impulsive hybrid controller as shown in Fig. 1 , where $C_{i_{k}} \in R^{n \times n}, k \in \mathbb{Z}$, are impulsive control gain matrices to be designed, and $\left\{t_{k}, k \in\right.$ $\mathbb{Z}\}$ are the impulsive instances satisfying $0 \leq t_{0}<t_{1}<t_{2}<$ $\cdots$, with $\sup _{k \in \mathbb{Z}}\left\{t_{k+1}-t_{k}\right\}<\infty$ and $\lim _{k \rightarrow \infty} t_{k}=\infty$.

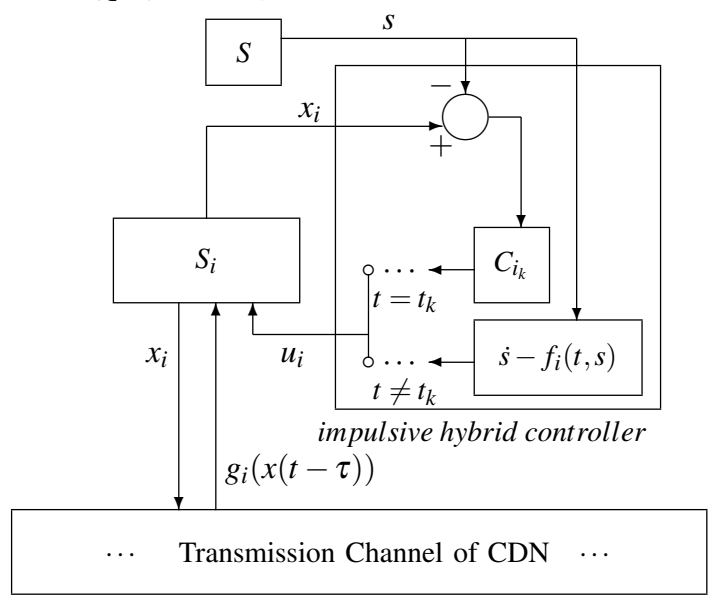

Fig.1. Impulsive consensus scheme of $S_{i}$.

Fig. 1 depicts the entire impulsive control scheme for the consensus ("impulsive consensus scheme" for short) between the known signal $s(t)$ and the CDN (1) with coupling timedelays, where $S_{i}$ stands for the $i$-th node, $S$ is objective vector function $s(t)$, and $g_{i}$ is the delay network coupling of the $i$ th node, $i=1,2, \cdots, N$. In this control scheme, the control signal is input into the CDN as: at impulsive instance $t_{k}$, the impulse signal $\left(C_{i_{k}}-I\right)\left(x_{i}\left(t_{k}\right)-s\left(t_{k}\right)\right)$ is input into the node $S_{i}$, and at other non-impulse times $t \neq t_{k}$, the signal $\dot{s}-f_{i}(t, s)$ containing the goal signal is input into the node $S_{i}$ of CDN.

By Fig.1, (4) is equivalent to the following system:

$$
\begin{gathered}
\dot{x}_{i}=f_{i}\left(t, x_{i}\right)+g_{i}+\dot{s}-f_{i}(t, s), \quad t \in\left[t_{k}, t_{k+1}\right), \\
\Delta x_{i}\left(t_{k}\right)=x\left(t_{k}\right)-x\left(t_{k}^{-}\right)=\left(C_{i_{k}}-I\right)\left(x_{i}\left(t_{k}\right)-s\left(t_{k}\right)\right), \\
t=t_{k}, k \in \mathbb{Z}, i=1,2, \cdots, N .
\end{gathered}
$$


Define the consensus errors as $e_{i}(t):=x_{i}(t)-s(t)$, then, by Assumptions 2.1-2.2, one has an error dynamical system:

$$
\begin{aligned}
& \dot{e}_{i}=A_{i} e_{i}+\hat{f}_{i}+\sum_{j=1}^{N} B_{i j} e_{j}\left(t-h_{i}\right)+\hat{g}_{i}, t \in\left[t_{k}, t_{k+1}\right), \\
& \Delta e_{i}=\left(C_{i_{k}}-I\right) e_{i}(t), t=t_{k}, k \in \mathbb{Z}, i=1, \cdots, N,
\end{aligned}
$$

where $\hat{f}_{i}=\hat{f}_{i}\left(t, x_{i}, s\right)=\tilde{f}_{i}\left(t, x_{i}\right)-\tilde{f}_{i}(t, s), \hat{g}_{i}=\hat{g}_{i}\left(x\left(t-h_{i}\right), s\right)=$ $\tilde{g}_{i}\left(\left(x_{1}\left(t-h_{i}\right), \cdots, x_{N}\left(t-h_{i}\right)\right)-\tilde{g}_{i}\left(s\left(t-h_{i}\right), \cdots, s\left(t-h_{i}\right)\right)\right.$.

Remark 2.1. It should be noticed that (1) represents a more general dynamical network than that considered in the literature in the following senses:

(i) The nodes in network (1) are non-identical, i.e., functions $f_{i}(i=1,2, \cdots, N)$ can be different. Moreover, if the given function $s(t)$ satisfies $\dot{s}=f_{i}(t, s), i=1,2, \cdots, N$, i.e., $s(t)$ is the same solution of single node, then, the impulsive consensus scheme is to make all states of the non-identical nodes approach the same solution $s(t)$. If all the functions $f_{i}$ are the same: $f_{i}=f$, and the given vector function $s(t)$ is a solution of system $\dot{y}=f(t, y)$, then the consensus problem is the synchronization problem discussed in the literature, for examples, see [20-27].

(ii) In the literature for study of complex dynamical networks, see examples [20-27], the coupling coefficient matrices have the form $B_{i j}=c C_{i j} \Gamma$, where $c>0$ denotes the coupling strength, $\Gamma=\operatorname{diag}\left\{r_{1}, r_{2}, \cdots, r_{n}\right\}$ and $C=\left(C_{i j}\right)_{N \times N}$, are the linking matrices of network nodes. However, only a single time delay $\tau$ is considered in the literature. In this paper, the multi time delays is involved.

(iii) The matrices and functions are considered as interval matrices and uncertain functions satisfying some conditions.

By [34], for any $X \in N\left[X_{1}, X_{2}\right]$, it can be formulated as:

$$
X=X_{0}+\Delta X=X_{0}+E \Sigma F,
$$

where $X_{0}=\frac{1}{2}\left(X_{1}+X_{2}\right), H=\frac{1}{2}\left(X_{2}-X_{1}\right)=\left(h_{i j}\right)_{n \times n}$, $E \cdot E^{T}=\operatorname{diag}\left\{\sum_{j=1}^{n} h_{1 j}, \cdots, \sum_{j=1}^{n} h_{n j}\right\}, \quad F^{T} \cdot F=$ $\operatorname{diag}\left\{\sum_{j=1}^{n} h_{j 1}, \cdots, \sum_{j=1}^{n} h_{j n}\right\}, \quad \Sigma \in \Sigma^{*}=\left\{\Sigma \in R^{n^{2} \times n^{2}}\right.$ : $\left.\Sigma=\operatorname{diag}\left(\varepsilon_{11}, \cdots, \varepsilon_{n n}\right),\left|\varepsilon_{i j}\right| \leq 1 ; i, j=1,2, \cdots, n\right\}$.

Assumption 2.3. For interval matrices $A_{i}, B_{i j}$, there exist known matrices $E, F_{A_{i}}, F_{i j}$, such that for any $\Sigma \in \Sigma^{*}$,

$$
\left[\Delta A_{i} \quad \Delta B_{i j}\right]=E \Sigma\left[\begin{array}{ll}
F_{A_{i}} & F_{i j}
\end{array}\right], i, j=1,2, \cdots, N .
$$

Definition 2.1. The error system (6) is said to be robustly globally asymptotically stable (RGAS) if, for any initial condition: $\phi \in C\left[\left[t_{0}-\tau, t_{0}\right], R^{n N}\right]$, for any $A_{i} \in N\left[A_{i_{1}}, A_{i_{2}}\right], B_{i j} \in$ $N\left[B_{i j_{1}}, B_{i j_{2}}\right]$, and for any $h_{j}$ with $0 \leq h_{j} \leq \tau$, the trivial solution of (6) is globally asymptotically stable (GAS).

Definition 2.2. The error system (6) is said to be robustly globally exponentially stable (RGES) with decay rate $\alpha$ if, for any initial condition: $\phi \in C\left[\left[t_{0}-\tau, t_{0}\right], R^{n N}\right]$, for any $A_{i} \in$ $N\left[A_{i_{1}}, A_{i_{2}}\right], B_{i j} \in N\left[B_{i j_{1}}, B_{i j_{2}}\right]$, and for any $h_{j}$ with $0 \leq h_{j} \leq \tau$, the trivial solution is globally exponentially stable (GES), i.e., for some positive numbers $\alpha>0, K>0$,

$$
\|e(t)\| \leq K\|\phi\|_{\tau} e^{-\alpha\left(t-t_{0}\right)}, \quad t \geq t_{0},
$$

where $\phi(t)=\left(\phi_{1}^{T}(t), \cdots, \phi_{N}^{T}(t)\right)^{T} \in R^{n N}, \phi_{i}(t) \in R^{n}$, and $\|\phi\|_{\tau}^{2}=\sum_{i=1}^{N}\left\|\phi_{i}\right\|_{\tau}^{2}$, with $\left\|\phi_{i}\right\|_{\tau}=\sup _{t_{0}-\tau \leq t \leq t_{0}}\left\{\left\|\phi_{i}(t)\right\|\right\}$.
Definition 2.3. The error system (6) is said to be quasiRGES with decay rate $\alpha$ if, for any initial condition: $\phi \in$ $C\left[\left[t_{0}-\tau, t_{0}\right], R^{n N}\right]$, for any $A_{i} \in N\left[A_{i_{1}}, A_{i_{2}}\right], B_{i j} \in N\left[B_{i j_{1}}, B_{i j_{2}}\right]$, and for any time-delays $h_{j}$ with $0 \leq h_{j} \leq \tau$, there exist two positive numbers: $\alpha>0, K>0$, such that

$$
\|e(t)\| \leq K\|\phi\|_{\tau} e^{-\alpha\left(k-t_{0}\right)}, \quad t \in\left[t_{k}, t_{k+1}\right), k \in \mathbb{Z} .
$$

Definition 2.4. The impulsive consensus scheme (5) is said to achieve global exponential impulsive consensus (GEIC) with speed rate $\alpha$ if, for any initial condition $\phi$, the error system (6) is RGES with decay rate $\alpha$. If the error system (6) is quasi-RGES with decay rate $\alpha$, then the network (1) is said to achieve quasi-GEIC with speed rate $\alpha$. If the system (6) is RGAS, then we say the network (1) can achieve global impulsive consensus (GIC).

Remark 2.2. (i) For the impulsive instances $\left\{t_{k}, k \in \mathbb{Z}\right\}, t_{k} \rightarrow$ $\infty$ if and only if $k \rightarrow \infty$; thus, Definition 2.3 is well-defined. (ii) In the GEIC or quasi-GEIC scheme, the consensus speed or consensus time can be estimated by using the speed rate, while in the GIC scheme it fails to do the estimation.

\section{GEIC CRITERIA}

In this section, GEIC criteria for network (1) will be established. Due to space limitations, we include only an outline for the results. The details are presented in [39].

By Assumption 2.3, we denote: $A=A_{0}+E_{A} \Sigma F_{A}$, and $B_{i j}=$ $B_{i j_{0}}+E \Sigma F_{i j}$, where $\Sigma \in \Sigma^{*}$.

Theorem 3.1. Let Assumptions 2.1-2.3 be satisfied. Suppose $\Delta_{\text {sup }} \triangleq \sup _{k \in \mathbb{Z}}\left\{t_{k}-t_{k-1}\right\}<\infty$ and that there exist positive definite matrices $P_{i} \in R^{n \times n}$ and constants $\varepsilon_{i j}>0, \tilde{\varepsilon}_{i j}>0, \varepsilon_{i}>$ $0, \hat{\alpha}_{i}>0, i, j=1,2, \cdots, N$, such that

(i) there exist positive constants $v_{i}>0, \mu_{i}>0$ such that

$$
v_{i} I \leq P_{i} \leq \mu_{i} I, \quad i=1,2, \cdots, N
$$

(ii) for $k \in \mathbb{Z}, i=1,2, \cdots, N$, the following LMIs hold:

$$
\left(\begin{array}{cccccc}
\Psi_{i}\left(A_{0}\right)-\hat{\alpha}_{i} P_{i} & P_{i} B_{i 1_{0}} & \cdots & P_{i} B_{i N_{0}} & P_{i} E & F_{A_{i}}^{T} \\
B_{i 1_{0}}^{T} P_{i} & -\varepsilon_{i 1} I & \cdots & 0 & 0 & F_{i 1}^{T} \\
\vdots & \vdots & \ddots & \vdots & \vdots & \vdots \\
B_{i N_{0}}^{T} P_{i} & 0 & \cdots & -\varepsilon_{i N} I & 0 & F_{i N}^{T} \\
E^{T} P_{i} & 0 & \cdots & 0 & -\varepsilon_{i}^{-1} I & 0 \\
F_{A_{i}} & F_{i 1} & \cdots & F_{i N} & 0 & -\varepsilon_{i} I
\end{array}\right)<0
$$

where $\quad \Psi_{i}\left(A_{0}\right)=P_{i} A_{0}+A_{0}^{T} P_{i}+2 L_{i} \sqrt{\frac{\mu_{i}}{v_{i}}} P_{i}+$ $\sum_{j=1}^{N} M_{i j} \tilde{\varepsilon}_{i j}^{-1}\left\|P_{i}\right\| I$

(iii) for any $k \in \mathbb{Z}$, the following inequality holds:

$$
\beta_{k} \triangleq \max _{1 \leq i \leq N}\left\{\lambda_{\max }\left(P_{i}^{-1} C_{i_{k}}^{T} P_{i} C_{i_{k}}\right)\right\}<1 ;
$$

(iv) there exists a positive integer $m \in \mathbb{Z}$ such that $t_{k-m} \leq$ $t_{k}-\tau<t_{k+1-m}$ for any $k \geq m, k \in \mathbb{Z}$, and the discrete system:

$$
z(k+1)=J_{k}(m) z(k), \quad k \in \mathbb{Z},
$$


is GES with decay rate $\sigma>0$, where

$J_{k}(m)=\left(\begin{array}{cccccc}0 & 1 & 0 & \cdots & 0 & 0 \\ 0 & 0 & 1 & \cdots & 0 & 0 \\ \vdots & \vdots & \vdots & \cdots & \vdots & \vdots \\ 0 & 0 & 0 & \cdots & 0 & 1 \\ \alpha_{k+1-m} & \alpha_{k+2-m} & \alpha_{k+3-m} & \cdots & \alpha_{k-1} & \tilde{\alpha}_{k}\end{array}\right)$

where $\tilde{\alpha}_{k-1}=\beta_{k} e^{p \Delta_{k}}+\alpha_{k-1}, \alpha_{k-j}=p_{2} \Delta_{k-j+1} e^{p \Delta_{k-j+1}}, j=$ $1,2, \cdots, m$, and $p=p_{1}+p_{2}, \quad p_{1}=\max _{1 \leq i \leq N}\left\{\hat{\alpha}_{i}\right\}, \quad p_{2}=$ $\max _{1 \leq i \leq N}\left\{\sum_{j=1}^{N} \frac{\hat{\varepsilon}_{j i}}{v_{i}}\right\}$, where $\hat{\varepsilon}_{i j}=\varepsilon_{i j}+M_{i j} \tilde{\varepsilon}_{i j}\left\|P_{i}\right\|$.

Then, the error system (6) is quasi-RGES with decay rate $\alpha \triangleq \frac{1}{2} \sigma$. Moreover, if there exist $k_{1} \geq k_{0}, k_{1} \in \mathbb{Z}$ and positive constant $\gamma>0$ such that $\sup _{k \geq k_{1}}\left\{\frac{t_{k}}{k}\right\} \leq \gamma$, then (6) is RGES with decay rate $\alpha \triangleq \frac{\sigma}{2 \gamma}$, and hence the CDN (1) can achieve GEIC with the given state $s(t)$ with speed rate $\alpha$.

Proof. Let Lyapunov-Krasovskii functional $V$ be: $V(e(t))=V_{1}(t)+V_{2}(t)$, where $V_{1}(t)=\sum_{i=1}^{N} e_{i}^{T}(t) P_{i} e_{i}(t)$, $V_{2}(t)=\sum_{i=1}^{N} \sum_{j=1}^{N} \hat{\varepsilon}_{j i} \int_{t-h_{j}}^{t} e_{i}^{T}(s) e_{i}(s) d s$, for some constants $\lambda_{i j}>0, i, j=1,2, \cdots, N$.

Claim 1: For $t \in\left[t_{k}, t_{k+1}\right), k \in \mathbb{N}$, by condition (i) and Schur Complement Theorem [43], we claim

$$
V(e(t)) \leq V\left(e\left(t_{k}\right)\right) e^{p\left(t-t_{k}\right)} .
$$

Claim 2: Let: $W(k)=\left(w_{1}(k), w_{2}(k), \cdots, w_{m}(k)\right)^{T}, k \in \mathbb{Z}$, where $w_{1}(k)=V\left(e\left(t_{k+1}\right)\right), w_{2}(k)=V\left(e\left(t_{k+2}\right)\right), \cdots, w_{m}(k)=$ $V\left(e\left(t_{k+m}\right)\right)$. Then, we claim $W(k-m) \leq z(k), \quad k \geq m-1$.

Claim 3: If the comparison system (13) is GES with decay rate $\sigma>0$, then we claim $\|e(t)\| \leq \hat{K}\|\phi\|_{\tau} e^{-\frac{\sigma}{2 \gamma}\left(t-t_{0}\right)}, t \geq t_{0}$, where $\hat{K}>0$ is some constant.

By Claims 1-3, we conclude that the result is true.

Corollary 3.1. Suppose that $\tau \leq t_{k}-t_{k-1}$ for any $k \in \mathbb{Z}$, i.e., $m=1$ in Theorem 3.1, and that there exist positive definite matrices $P_{i} \in R^{n \times n}$ and constants $\varepsilon_{i j}>0, \tilde{\varepsilon}_{i j}>0, \varepsilon_{i}>0, \hat{\alpha}_{i}>$ $0, i, j=1,2, \cdots, N, k \in \mathbb{Z}$, such that (i)-(iii) of Theorem 3.1 hold, while (iv) of Theorem 3.1 is replaced by:

(iv*) there exists a positive constant $\sigma>0$ such that

$$
\ln \left(\beta_{k}+p_{2} \tau\right)+(p+\sigma)\left(t_{k}-t_{k-1}\right) \leq 0,
$$

where $\beta_{k}, p_{1}, p_{2}, p$ are defined in Theorem 3.1.

Then, the error system (6) is RGES with decay rate $\alpha \triangleq \frac{\sigma}{2}$ and thus the CDN (1) can achieve GEIC with the given state $s(t)$ with speed rate $\alpha$.

Remark 3.1. From Corollary 3.1, if $\tau \leq t_{k}-t_{k-1}$ for any $k \in \mathbb{Z}$, we get the maximum estimations of time-delay $\tau^{*}$ and the interval of impulses as follows:

$$
\begin{aligned}
& \tau^{*} \leq \sup _{k \in \mathbb{Z}}\left\{\frac{e^{-(p+\sigma)\left(t_{k}-t_{k-1}\right)}-\beta_{k}}{p}\right\}, \\
& \Delta_{\text {sup }} \leq \sup _{k \in \mathbb{Z}}\left\{\frac{-\ln \left(\beta_{k}+p_{2} \tau\right)}{p+\sigma}\right\} .
\end{aligned}
$$

Corollary 3.2. Suppose $\Delta_{\text {sup }} \triangleq \sup _{k \in \mathbb{Z}}\left\{t_{k}-t_{k-1}\right\}<\infty$ and that there exist positive definite matrices $P_{i} \in R^{n \times n}$ and constants $\varepsilon_{i j}>0, \hat{\varepsilon}_{i j}>0, \varepsilon_{i}>0, \hat{\alpha}_{i}>0, i, j=1,2, \cdots, N$, such that (i)-(iii) of Theorem 3.1 hold, while (iv) of Theorem 3.1 is replaced by (iv**) there exists a positive integer $m>1$ such that $t_{k-m} \leq$ $t_{k}-\tau<t_{k+1-m}$ for any $k \geq m, k \in \mathbb{Z}$, and

$$
J(m) \triangleq\left(\begin{array}{cccccc}
0 & 1 & 0 & \cdots & 0 & 0 \\
0 & 0 & 1 & \cdots & 0 & 0 \\
\vdots & \vdots & \vdots & \cdots & \vdots & \vdots \\
0 & 0 & 0 & \cdots & 0 & 1 \\
a & a & a & \cdots & a & a+b
\end{array}\right)
$$

satisfies the spectrum radius of matrix $J(m)$ conditions: $\rho(J(m))<e^{-\sigma}$, where $\sigma>0$ is some positive constant, $a=$ $p_{2} \Delta_{\text {sup }} e^{p \Delta_{\text {sup }}}, b=\beta e^{p \Delta_{\text {sup }}}, \beta=\sup _{k \in \mathbb{Z}}\left\{\lambda_{\max }\left(P_{i}^{-1} C_{i_{k}}^{T} P_{i} C_{i_{k}}\right)\right\}$, and $p, p_{1}, p_{2}$ are defined in Theorem 3.1.

Then, the error system (6) is quasi-RGES with decay rate $\alpha \triangleq \frac{1}{2} \sigma$. Moreover, if there exist $k_{1} \geq k_{0}, k_{1} \in \mathbb{Z}$ and positive constant $\gamma>0$ such that $\sup _{k \geq k_{1}}\left\{\frac{t_{k}}{k}\right\} \leq \gamma$, then (6) is RGES with decay rate $\alpha \triangleq \frac{\sigma}{2 \gamma}$, and hence the CDN (1) can achieve GEIC with the given state $s(t)$ with speed rate $\alpha$.

\section{Impulsive Consensus Control Design}

In this section, by using the obtained results, we design impulsive control gain matrices for the CDN (1) such that GEIC can be achieved.

Theorem 4.1. Assume $\Delta_{\text {sup }} \triangleq \sup _{k \in \mathbb{Z}}\left\{t_{k}-t_{k-1}\right\}<\infty$ and that conditions (i)-(ii) of Theorem 3.1 still hold, while conditions (iii)-(iv) are changed to the following (iii')-(iv'):

(iii') there exist positive constants $0<\beta_{i}<1, i=$ $1,2, \cdots, N$, such that the following LMIs hold:

$$
\left(\begin{array}{ccc}
\Omega_{1} & \Omega_{2} & Y_{i}^{T} \\
\Omega_{2}^{T} & -I & 0 \\
Y_{i} & 0 & -P_{i}
\end{array}\right) \leq 0
$$

where $\Omega_{1}=P_{i}+Y_{i}^{T}+Y_{i}-\beta_{i} P_{i}, \Omega_{2}=P_{i} E+Y_{i}^{T} E$;

(iv') let $\beta_{k}=\max _{1 \leq i \leq N}\left\{\beta_{i}\right\}, k \in \mathbb{Z}$, then the condition (iv) of Theorem 3.1 holds.

Then, under impulsive control gain matrices $\left\{C_{i_{k}}=I+\right.$ $\left.P_{i}^{-1} Y_{i}, i=1,2, \cdots, N, k \in \mathbb{Z}\right\}$, the error system (6) is quasiRGES with decay rate $\alpha=\frac{1}{2} \sigma$. Moreover, if there exist $k_{1} \geq$ $k_{0}, k_{1} \in \mathbb{Z}$ and positive constant $\gamma>0$ such that $\sup _{k \geq k_{1}}\left\{\frac{t_{k}}{k}\right\} \leq$ $\gamma$, then system (6) is RGES with decay rate $\alpha \triangleq \frac{\sigma}{2 \gamma}$, and thus the CDN (1) can be achieved GEIC with the given state $s(t)$ with speed rate $\alpha$.

Corollary 4.1. Assume the conditions (i)-(ii) of Theorem 3.1 and condition (iii*) of Theorem 4.1 hold. Then, under impulsive control gain matrices $\left\{C_{i_{k}}=I+P_{i}^{-1} Y_{i}, i=1,2, \cdots, N, k \in\right.$ $\mathbb{Z}\}$, condition (iv*) implies that the CDN (1) can achieve GEIC with speed rate $\frac{\sigma}{2}$; condition (iv**) implies that the CDN (1) can achieve GEIC with speed rate $\frac{\sigma}{2}$; and (iv***) implies that (6) is RGAS and the CDN (1) can achieve GIC.

In the following, one example is given for illustration.

Example 4.1. Use the chaotic Colpitts' oscillator as nodes of the CDN. The Colpitts' oscillator is described by:

$$
\dot{y}=A_{0} y+\varphi(y)
$$

where $y=\left(\begin{array}{l}y_{1} \\ y_{2} \\ y_{3}\end{array}\right), A_{0}=\left(\begin{array}{ccc}0 & \alpha & 0 \\ -\sigma & -\gamma \sigma & -\sigma \\ a_{1} \beta & \beta & 0\end{array}\right)$, and $\varphi(y)=$ $\left(0,0, a_{3} \beta y_{1}^{3}\right)^{T}$, in which $\alpha, \beta, \sigma, a_{1}, \gamma, a_{3} \in R$. It is known that 
with parameters $\alpha=2.4, \beta=2.2, \sigma=1, \gamma=0.252, a_{1}=1$, and $a_{3}=-0.2$, the oscillator (20) is chaotic.

Suppose that the CDN (1) is given by

$$
\dot{x}_{i}=A_{i} x_{i}+\varphi\left(x_{i}\right)+\sum_{j=1}^{N} B_{i j} x_{j}\left(t-h_{i}\right), i=1, \cdots, N,
$$

where $x_{i}=\left(x_{i 1}, x_{i 2}, x_{i 3}\right)^{T}$, with matrix $A_{i} \in N[\underline{A}, \bar{A}]$, where

$$
\underline{A}=\left(\underline{a}_{i j}\right)_{3 \times 3}=\left(\begin{array}{ccc}
-0.5 & \alpha-0.5 & -0.5 \\
-\sigma-0.5 & -\gamma \sigma-0.5 & -\sigma-0.5 \\
a_{1} \beta-0.5 & \beta-0.5 & -0.5
\end{array}\right),
$$

$\bar{A}=\left(\underline{a}_{i j}+1\right)_{3 \times 3} ;$ and $B_{i j}$ satisfy: $B_{i j}=B_{i j_{1}}=B_{i j_{2}}$, $i, j=1,2, \cdots, N, \quad B_{i i}=\left(\begin{array}{ccc}0.5 & 0.5 & 0 \\ 0 & 0.5 & 0.2 \\ 0 & 0 & -0.5\end{array}\right), \quad B_{i, i+1}=$ $\left(\begin{array}{ccc}-1.0 & -0.3 & 0 \\ 0 & 0.25 & -0.1 \\ 0 & 0 & 1.0\end{array}\right), B_{i, i+2}=\left(\begin{array}{ccc}0.5 & -0.2 & 0 \\ 0 & -0.75 & -0.1 \\ 0 & 0 & -0.5\end{array}\right)$, $B_{N-1, N+1}=B_{N-1,1}, \quad B_{N-1, N+2}=N_{N-1,2}, \quad B_{N, N+1}=B_{N 1}$, $B_{N, N+2}=B_{N 2}$.

Suppose the goal $s(t)$ is the solution of Lorenz system:

$$
\dot{s}=L s+\tilde{\varphi}(s)
$$

where $s=\left(\begin{array}{l}s_{1} \\ s_{2} \\ s_{3}\end{array}\right), \quad L=\left(\begin{array}{ccc}-b_{1} & b_{1} & 0 \\ b_{2} & -1 & 0 \\ 0 & 0 & -b_{3}\end{array}\right), \quad \varphi(s)=$ $\left(0,-s_{1} s_{3}, s_{1} s_{2}\right)^{T}$, in which $b_{1}, b_{2}, b_{3} \in R$. It is well-known that with parameters $b_{1}=10, b_{2}=28, b_{3}=\frac{8}{3}$, the Lorenz system (22) is chaotic.

It is easy to show for any matrix $A \in N[\underline{A}, \bar{A}], A$ is not a stable matrix. In the following, by using Theorem 3.1 , we design impulsive control gain matrices $K_{i_{k}}-I, i=$ $1, \cdots, 10, k \in \mathbb{Z}$ and the impulsive instances $t_{k}, k \in \mathbb{Z}$ such that the system in form of (4) can achieve GEIC.

By [42], we get that $\left\|\frac{\partial \varphi(y)}{\partial y}\right\| \leq 5.28$, which implies that $L_{i}=5.28, \quad i=1,2, \cdots, 10$. Choosing $\varepsilon_{i j}=$ $1, \varepsilon_{i}=1, \quad v_{i}=1, \mu_{i}=2$, we solve the LMIs in (ii) of Theorem 3.1, for $i=1,2, \cdots, 10$, getting $\hat{\alpha}_{i}=6$, and $P_{i}=\left(\begin{array}{lll}1.5437 & 0.3646 & 0.1371 \\ 0.3646 & 1.4298 & 0.1290 \\ 0.1371 & 0.1290 & 1.1520\end{array}\right)$. Then, by solving the LMIs in (iii') of Theorem 4.1, we get that $\beta_{k}=$ 0.01 , and $Y_{i}=\left(\begin{array}{lll}-1.5328 & -0.3546 & -0.1330 \\ -0.3546 & -1.4215 & -0.1264 \\ -0.1330 & -0.1264 & -1.1502\end{array}\right)$. Thus, we get $p_{1}=6, p_{2}=10, \beta_{k}=0.01, \quad K_{i_{k}}-I=P_{i}^{-1} Y_{i}=$ $\left(\begin{array}{ccc}-0.9944 & 0.0054 & 0.0024 \\ 0.0054 & -0.9957 & 0.0012 \\ 0.0023 & 0.0011 & -1.0004\end{array}\right)$. Moreover, there exists $\sigma=0.01$ such that (19) in Corollary 3.1 holds. By Remark 3.2 , we get $\tau^{*}<0.0619$ and $\Delta_{\text {sup }} \leq 0.1107$. Thus, we can design the impulsive controllers $\left\{K_{i_{k}} x_{i}, t_{k}\right\}$ as:

Case 1: If $\tau \leq t_{k}-t_{k-1}, k \in \mathbb{Z}$, then, let: $t_{0}=0, t_{k}-$ $t_{k-1}=0.1, k \in \mathbb{Z}$, and $K_{i_{k}}, i=1,2, \cdots, 10$, are chosen as above. Therefore, by Theorem 4.1, the impulsive controllers $\left\{K_{i_{k}} e_{i}, t_{k}\right\}$ can achieve GEIC for all node states $x_{i}(t)(i=$ $1,2, \cdots, 10)$ and the dynamical goal $s(t)$. Moreover, the consensus speed rate is $\alpha=\frac{1}{2} \sigma=0.005$.

Case 2: If there is $m>1$ such that $t_{k-m} \leq t_{k}-\tau<t_{k+1-m}, k \in$ $\mathbb{Z}$, then, by Corollary 3.2 , we design the impulsive controllers. For example, let $\tau=h_{i}=0.05$, and $m=2$, then by Corollary 3.2, the impulsive instances can be set as: $t_{0}=0, t_{k}-t_{k-1}=0.03, k \in \mathbb{Z}$. Therefore, by Corollary 3.2, the impulsive controllers $\left\{K_{i_{k}} e_{i}, t_{k}\right\}$ can achieve GEIC for all node states $x_{i}(t)(i=1,2, \cdots, 10)$ and the dynamical goal $s(t)$. Moreover, the consensus speed rate is $\alpha=\frac{1}{2 \gamma} \sigma=$ 0.0045 .

In simulation, let $\tau=h_{i}=0.05, t_{k+1}-t_{k}=0.03, k \in \mathbb{Z}$, and, without loss of generality, $x_{i}(t)=0$ whenever $t<0$, and $x_{i}(0) \neq 0, i=1,2, \cdots, N$. The matrices $A_{i} \in N[\underline{A}, \bar{A}]$ are set as: $A_{1}=A_{2}=A_{3}=\tilde{A}_{1}, A_{4}=A_{5}=A_{6}=\tilde{A}_{2}, A_{7}=A_{8}=\tilde{A}_{3}$, and $A_{9}=A_{10}=\tilde{A}_{4}$, where $\tilde{A}_{2}=A_{i_{0}}, \tilde{A}_{3}=\underline{A}, \tilde{A}_{4}=\bar{A}$, where $\tilde{A}_{1}=\underline{A}+\operatorname{Rand}(3,3), \tilde{A}_{i_{0}}=A_{0}$.

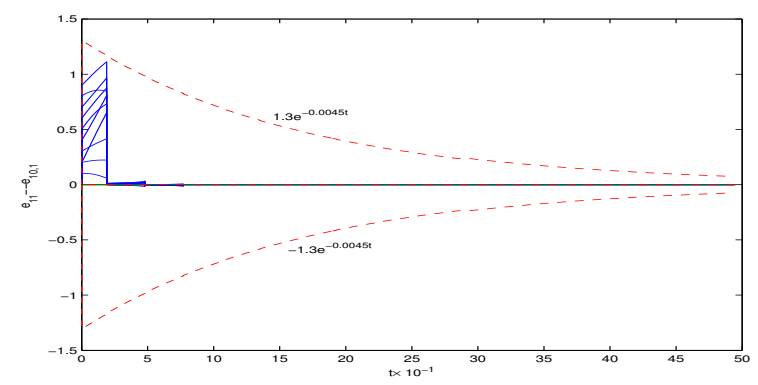

Fig.2. Exponential consensus error properties (with speed rate $0.0045)$ of $e_{k 1}(t), k=1,2, \cdots, 10$.

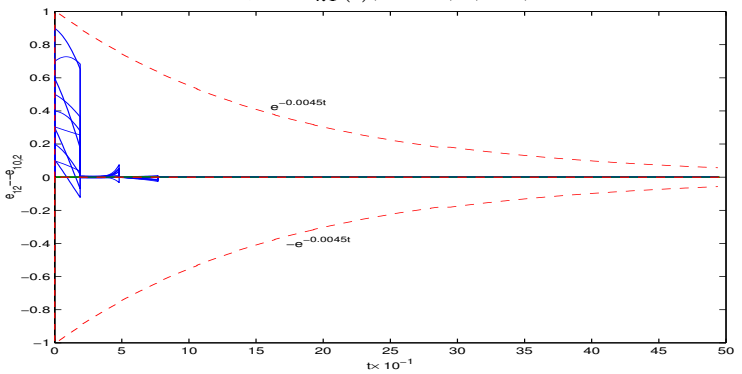

Fig.3. Exponential consensus error properties (with speed rate $0.0045)$ of $e_{k 2}(t), k=1,2, \cdots, 10$.

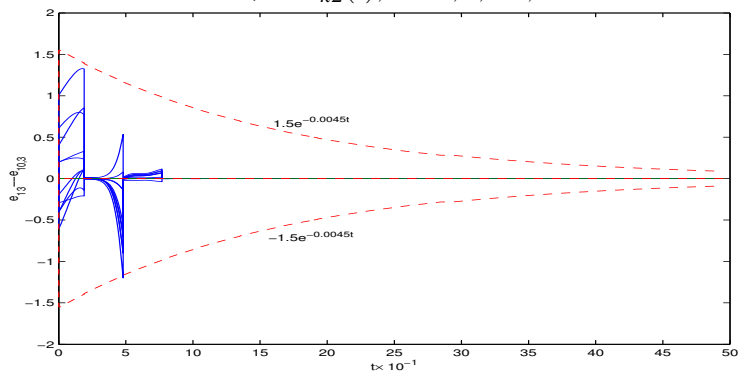

Fig.4. Exponential consensus error properties (with speed rate $0.0045)$ of $e_{k 3}(t), k=1,2, \cdots, 10$. 


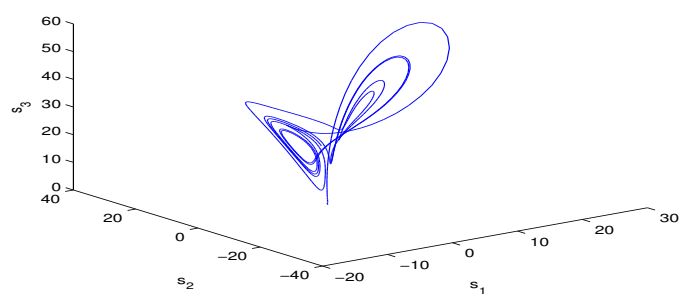

Fig.5. The phase figure of Lorenz system.

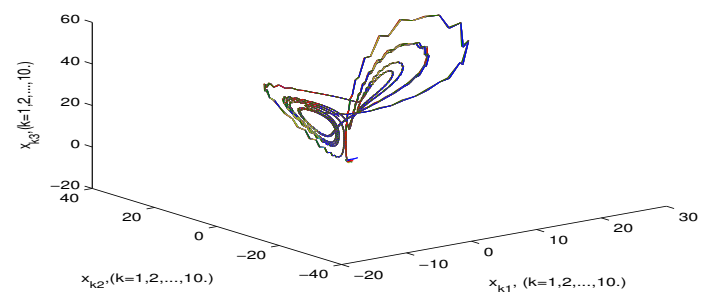

Fig.6. The phase figure of the network under impulsive control.

In Figs. 2-4, one can see the consensus properties of the goal state $s_{i}$ of the Lorenz system (22) and node states $x_{l i}$, $l=1,2, \cdots, 10, i=1,2,3$, of the network. In Figs.5-6, one can see the whole consensus properties of Lorenz system (22) and the network with coupling delay $\tau$.

\section{REFERENCES}

[1] L. M. Pecora and T. L. Carroll, "Synchronization in chaotic systems," Phys. Rev. Lett., 64(8), 1990, pp.821-824.

[2] T. L. Carroll and L. M. Pecora, "Synchronization in chaotic circuits," IEEE Trans. on Circuits Syst.-I, 38(4), 1991, pp.453-456.

[3] L. Kocarev and U. Parlitz, "General approach for chaotic synchronization with application to communication," Phys. Rev. Lett., 74, 1995 , pp.5028-5031.

[4] C. W. Wu, T. Yang, and L. O. Chua, "On adaptive synchronization and control of nonlinear dynamical systems," Int. J. Bifurcation Chaos, 6 , 1996, pp.455-471.

[5] G. Grassi and S. Mascolo, "Nonlinear observer design to synchronize hyperchaotic systems vis a scalar signal," IEEE Trans. on Circuits Syst., 44, 1997, pp.1011-1014.

[6] L. Kocarev, G. M. Maggio, M. Ogorzalek, L. Pecora, and K. Yao, "Introduction to the special issue," IEEE Trans. on Circuits Syst.-I, 48, 2001, pp.1385-1386.

[7] G. Chen and X. Dong, "On feedback control of chaotic continuoustime systems," IEEE Trans. on Circuits Syst., 40, 1993, pp.591-601.

[8] M. E. Yalcin, J. A. K. Suykens, and J. Vandewalle, "Master-slave synchronization of Lur'e systems with time-delay," Int. J. Bifurcation Chaos, 11, 2001, pp.1707-1772.

[9] T. Yang and L. O. Chua, "Impulsive stabilization for control and synchronization of chaotic systems: Theory and application to secure communication," IEEE Trans. on Circuits Syst.-I, 44, 1997, pp.976988.

[10] H. M. Rodrigues, L. F. C. Alberto, and N. G. Bretas, "On the invariance principle: Generalizations and applications to synchronization", IEEE Trans. on Circuits Syst.-I, 47(5), 2000, pp.730-738.

[11] A. Khadra, X. Liu, and X. Shen, "Application of impulsive synchronization to communication security," IEEE Trans. on Circuits Syst.-I, 50, 2003, pp.341-351.

[12] L. M. Pecora and T. L. Carroll, "Master stability functions for synchronized coupled systems," Phys. Rev. Lett., 80(10), 1998, pp.2109-2112.

[13] L. M. Pecora, T. L. Carroll, G. Johnson, D. Mar, and K. S. Fink, "Synchronization stability in coupled oscillator arrays: solution for arbitrary configurations," Int. J. Bifurcation Chaos, 10(2), 2000, pp.273-290.
[14] C. W. Wu and L. O. Chua, "Synchronization in an array of linearly coupled dynamical systems," IEEE Trans. on Circuits Syst.-I, 42(8), 1995, pp.430-447.

[15] C. W. Wu, "Perturbation of coupling matrices and its effect on the synchronizability in arrays of coupled chaotic systems," Phys. Lett. A, 319, 2003, pp.495-503.

[16] X. Wang and G. Chen, "Synchronization in small-world dynamical networks," Int. J. Bifurcation Chaos, 12(1), 2002, pp.187-192.

[17] X. Wang and G. Chen, "Synchronization in scale-free dynamical networks: Robustness and Fragility," IEEE Trans. on Circuits Syst.I, 49(1), 2002, pp.54-62.

[18] J. Jost and M. P. Joy, "Spectral properties and synchronization in coupled map lattices," Phys. Rev. E, 65(016201), 2002.

[19] P. M. Grade and C. K. Hu, "Synchronization chaos in coupled map lattices with small-world interactions," Phys. Rev. E, 62(5), 2000, pp.6409-613.

[20] Z. Li and G. Chen, "Robust adaptive synchronization of uncertain dynamical networks," Phys. Lett. A, 324, 2004, pp.166-178.

[21] G. Chen, J. Zhou, and Z. Liu, "Global synchronization of coupled delayed neural networks and applications to chaotic CNN models," Int. J. Bifurcation Chaos, 14(7), 2004, pp.2229-2240.

[22] C. Li and G. Chen, "Synchronization in general complex dynamical networks with coupling delays," Phys. A, 343, 2004, pp.263-278.

[23] C. Li, H. Xu, X. Liao and J. Yu, "Synchronization in small-world oscillator networks with coupling delays," Phys. A, 335, 2004, pp.359364.

[24] W. Lu and T. Chen, "Synchronization analysis of linearly coupled networks of discrete time systems," Phys. D, 198, 2004, pp.148-168.

[25] J. L $\ddot{u}$ and G. Chen, "A time-varying complex dynamical model and its controlled synchronization criteria," IEEE Trans. Automat. Contr., 50(6), 2005, pp.841-846

[26] G.P. Jiang, W.K.S. Tang, and G. Chen, "A state-observer-based approach for synchronization in complex dynamical networks", IEEE Trans. on Circuits Syst.-I, 53(12), 2006, pp.2739-2745.

[27] J. Xiang, and G. Chen, "On the V-stability of complex dynamical networks", Automatica, 43, 2007, pp.1049-1057.

[28] V. Lakshmikantham, D. D. Bainov, and P. S. Simeonov, Theory of impulse differential equations. Singapore World Scientific, 1989.

[29] D. D. Bainov, and P. S. Simeonov, Systems with impulsive effects: Stability theory and applications. New York: Halsted Press, 1989.

[30] H. Ye, A. N. Michel, and L. Hou, "Stability analysis of systems with impulsive effects," IEEE Transactions on Automatic Control, 43(12), 1998, pp. 1719-1723.

[31] T. Yang, Impulsive Control Theory, Springer-Verlag, 2001.

[32] Z. G. Li, Y. C. Soh, C. Y. Wen, Switched and Impulsive Systems. Analysis, Design and Application. Springer-Verlag, April, 2005.

[33] Z. G. Li, C. Y. Wen, Y. C. Soh, "Analysis and design of impulsive control systems," IEEE Trans. Automat. Control, 46, 2001, pp.894899 ,

[34] B. Liu, X. Z. Liu, G. Chen, and H. Wang, "Robust impulsive synchronization of uncertain dynamical networks," IEEE Trans. on Circuits Syst.-I. Regular Paper, 52(7), 2005, pp. 1431-1441.

[35] B. Liu, X. Liu, and K. L. Teo, "Razumikhin-type theorems on exponential stability of impulsive delay systems," IMA J. Appl. Math., 71, 2006, pp.47-61.

[36] B. Liu, H. J. Marquez, "Razumikhin-type stability theorems for discrete delay systems", Automatica, 43(7), 2007, pp.1219-1225.

[37] B. Liu, David J. Hill, "Robust stability of complex impulsive dynamical systems", Proc. 46th IEEE Conf. Decision Control, New Orleans, USA, 2007, pp.103-108

[38] B. Liu, David J. Hill, "Impulsive tracking and consensus control for time-delay complex dynamical networks with non-identical nodes", in preparation.

[39] Z. H. Guan, D. J. Hill, and X. Shen, "On hybrid impulsive and switching systems and applications to nonlinear control", IEEE Trans. Automat. Control, 50, 2005, pp.1058-1062.

[40] J. K. Hale, S. M. Verduyn Lunel. Introduction to functional differential equations. New York: Springer Press, 1993.

[41] A. Isidori, Nonlinear control systems II. London: Springer, 1999.

[42] G. Chen, Controlling Chaos and Bifurcations in Engineering Systems. Boca Raton, FL: CRC Press, 2000.

[43] S. Boyd, L. El Ghaoui, E. Feron, and V. Balakrishnan, Linear matrix inequality in systems and control theory. SIAM, Philadelphia, 1994. 